EPJ Web of Conferences 60, 12010 (2013)

DOI: $10.1051 /$ epjconf/20136012010

(C) Owned by the authors, published by EDP Sciences, 2013

\title{
Higgs boson production in the SM and MSSM to NNLO and beyond
}

\author{
Matthias Steinhauser ${ }^{1, a}$ \\ ${ }^{1}$ Karlsruhe Institute of Technology (KIT), Institut für Theoretische Teilchenphysik
}

\begin{abstract}
In this contribution a brief review about the status of higher order corrections to Higgs boson production within the Minimal Supersymmetric Standard Model (MSSM) is given. Furthermore the first activities towards third-order corrections in the Standard Model (SM) are discussed.
\end{abstract}

\section{Introduction}

The discovery of a new Higgs boson-like particle at LHC [1, 2] has triggered plenty activities with the aim to pin down its properties like couplings and decay rates. In this contribution we consider the production cross section of a Higgs boson in the gluon-fusion channel and discuss the status both for the SM and the MSSM.

The theoretical framework of our calculations is the effective theory where all particles which are heavier than the Higgs boson are integrated out. This leads to an effective Higgs-gluon interaction which is described by

$$
\mathcal{L}_{\mathrm{eff}}=-\frac{H}{v} C_{1} \frac{1}{4} G_{\mu \nu} G^{\mu v},
$$

where $G_{\mu \nu}$ is the gluon field strength tensor and $C_{1}$ is the coupling (or matching coefficient) containing the remnant dependence on the heavy degrees of freedom. Within the SM $C_{1}$ only depends on the top quark mass via $\ln \left(\mu^{2} / m_{t}^{2}\right)$ where $\mu$ is the renormalization scale. In the MSSM, $C_{1}$ becomes a complicated function of all heavy mass scales and $\mu$.

In Refs. [3-6] it has been demonstrated that at nextto-next-to-leading order (NNLO) the effective-theory approach of Eq. (1) approximates the exact SM result with an accuracy below $1 \%$, in particular for Higgs boson masses around $126 \mathrm{GeV}$. Numerical NLO calculations [7] suggest a similar behaviour in the MSSM.

In Section 2 NNLO SUSY QCD corrections are considered within the MSSM. Afterwards, we summarize in Section 3 the first steps towards $\mathrm{N}^{3} \mathrm{LO}$ in the SM.

\section{NNLO corrections to $g g \rightarrow H+X$ in the MSSM}

The computation of higher order supersymmetric corrections within the effective-theory framework requires the evaluation of loop corrections to the matching coefficient $C_{1}$. Several groups have computed two-loop corrections

\footnotetext{
a e-mail: matthias.steinhauser@kit.edu
}
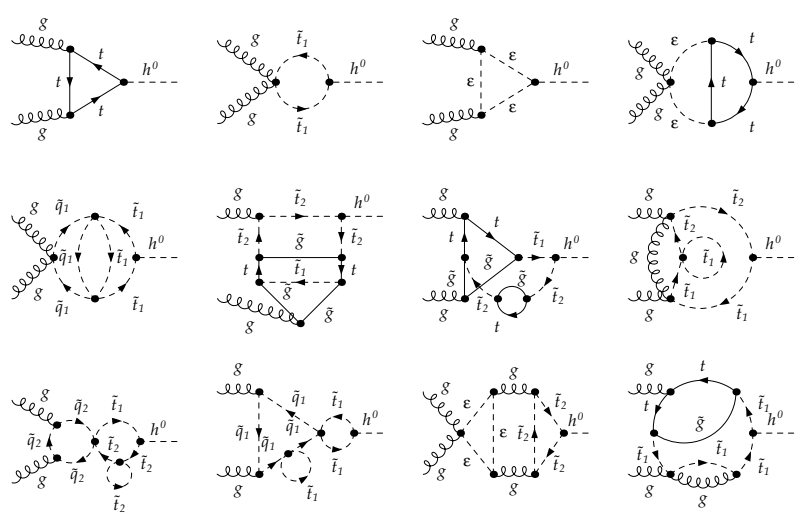

Figure 1. Feynman diagrams contributing to $C_{1}$.

both in the top [8-10] and bottom sector [7, 11, 12]. NLO calculations in the full theory have been performed in Ref. [7]. Building blocks for a (semi) analytic fulltheory calculation have been provided in Refs. [13, 14]; a complete calculation along these lines is still missing. Recently the effective-theory NLO corrections have been implemented in the publicly available computer code SusHi [15]. At NNLO the rough approximation of Ref. [16] has been implemented, i.e., the genuine SUSY corrections to $C_{1}$ have been set to zero at three loops.

In this contribution we want to discuss the numerical effect of three-loop corrections to $C_{1}$ which are needed in order to obtain a complete NNLO prediction of the Higgs boson production cross section. Sample diagrams contributing to $C_{1}$ at one, two and three loops are shown in Fig. 1. The symbols $t, \tilde{t}_{i}, g, \tilde{g}, h$ and $\varepsilon$ denote top quarks, top squarks, gluons, gluinos, Higgs bosons and $\varepsilon$ scalars, respectively. ${ }^{1}$ For the computation of $C_{1}$ it is possible to expand the Feynman integral in the external gluon momenta which leads to vacuum diagrams. In contrast to the $\mathrm{SM}$, in the MSSM many different mass scales are present

\footnotetext{
${ }^{1} \varepsilon$ scalars are auxiliary particles introduced to implement regularization by dimensional reduction [17] which respects supersymmetry.
} 
which increases the complexity of the calculation significantly. In fact, the currently available tools do not allow for an exact calculation and one has to rely on approximation methods. In Refs. $[18,19]$ hierarchies in the occurring masses have been defined in such a way that phenomenological interesting scenarios can be studied. Furthermore, sophisticated expansion schemes have been defined which select the representation of the perturbative series with largest radius of convergence. Moreover, reliable error estimates for each point in the parameter space are obtained. In this way three-loop corrections to $C_{1}$ have been evaluated for the top and bottom sector, neglecting, however, the bottom Yukawa coupling. In a first step a simplified scenario where all supersymmetric masses are identical has been considered in [20]. In Ref. [18] the results have been generalized by considering various hierarchies of the involved supersymmetric particle masses. Furthermore, details on the renormalization procedure and the treatment of evanescent couplings are discussed. The results of [18] have been cross-checked in Ref. [19] where a low-energy theorem has been used in order to obtain $C_{1}$ from the decoupling constant of $\alpha_{s}$.

In order to incorporate all know results in the final prediction for the cross section we apply the following formula

$$
\begin{aligned}
& \sigma(p p \rightarrow h+X)=\left(1+\delta^{\mathrm{EW}}\right) \times \\
& {\left[\left.\sigma_{t b}^{\mathrm{SQCD}}\left(\mu_{s}\right)\right|_{\mathrm{NLO}}-\left.\sigma_{t}^{\mathrm{SQCD}}\left(\mu_{s}\right)\right|_{\mathrm{NLO}}\right.} \\
& \left.+\left.\sigma_{t}^{\mathrm{SQCD}}\left(\mu_{s}, \mu_{h}\right)\right|_{\mathrm{NNLO}}\right]
\end{aligned}
$$

where $\sigma_{t b}$ refers to the NLO result including all top and bottom effects. After subtracting the top quark/top squark contributions with the help of $\left.\sigma_{t}^{\mathrm{SQCD}}\left(\mu_{s}\right)\right|_{\mathrm{NLO}}$ we can add the result from the top quark/top squark up to NNLO. ${ }^{2}$ Finally, electroweak effects are taken into account in a multiplicative way.

In Fig. 2 we discuss numerical effects of the individual terms in Eq. (2) using the $m_{h}^{\max }$ scenario of Ref. [21] as a basis. We apply slight modifications which lead to the following parameters (see Ref. [18] for explanations of the parameters)

$$
\begin{array}{r}
A_{b}=A_{\tau}=2469.48 \mathrm{GeV}, \quad A_{t}=1500 \mathrm{GeV}, \\
M_{1}=5 s_{W}^{2} /\left(3 c_{W}^{2}\right) M_{2}, \quad M_{2}=200 \mathrm{GeV}, \\
M_{3}=800 \mathrm{GeV}, \quad M_{A}=1000 \mathrm{GeV}, \\
\mu_{\text {susy }}=200 \mathrm{GeV}, \quad m_{\text {susy }}=1000 \mathrm{GeV}, \\
\tan \beta=20 .
\end{array}
$$

In addition we have the parameter $\tilde{m}_{t}$, the singlet soft SUSY breaking parameter of the right-handed top squark, which is varied in Fig. 2 . The default value $\tilde{m}_{t}=400 \mathrm{GeV}$ in combination with SOFTSUSY [22] leads to the following

\footnotetext{
${ }^{2}$ Note that $\sigma_{t}$ also contains contributions from a non-vanishing Higgsbottom squark coupling, see Ref. [18] for details. Since they are small we refer in Eq. (2) only to the top quark/top squark sector.
}

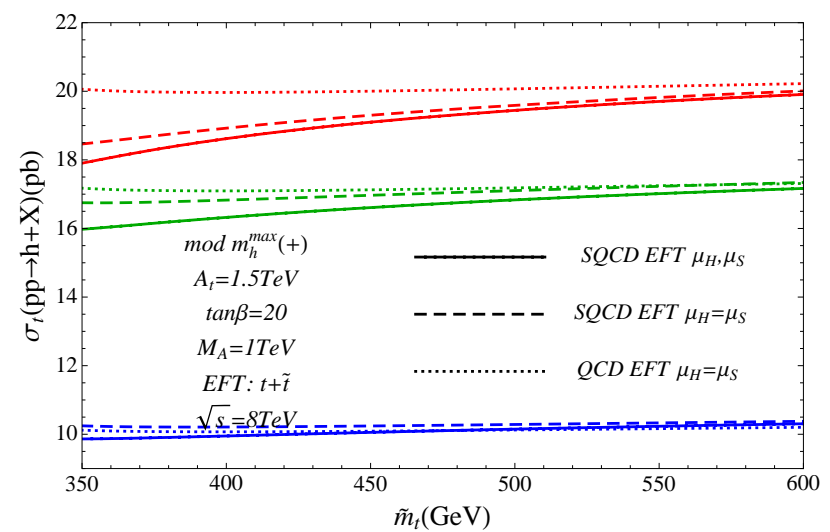

(a)

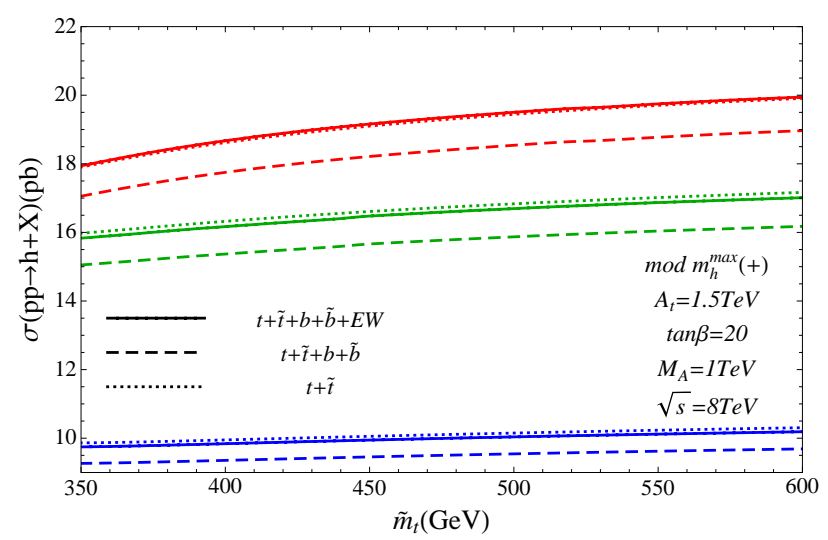

(b)

Figure 2. Cross section as a function of the singlet soft SUSY breaking parameter of the right-handed top squark, $\tilde{m}_{t}$. (a) top quark/top squark contribution $\sigma_{t}$. (b) complete contribution including also bottom quark and electroweak effects as described in Eq. (2).

values for the $\overline{\mathrm{DR}}$ masses

$$
\begin{array}{lr}
m_{\tilde{t}_{1}}=370 \mathrm{GeV}, & m_{\tilde{t}_{2}}=1045 \mathrm{GeV}, \\
m_{\tilde{q}}=1042 \mathrm{GeV}, & m_{\tilde{g}}=860 \mathrm{GeV},
\end{array}
$$

where $m_{\tilde{q}}$ corresponds to the average of $m_{\tilde{u}}, m_{\tilde{d}}, m_{\tilde{s}}, m_{\tilde{c}}$ and $m_{\tilde{b}}$ and the renormalization scale has been set to the on-shell top quark mass.

In Ref. [18] the program H3m [23, 24] has been used in order to compute the lightest MSSM Higgs boson mass. Combining H3m with version 2.6.5 of FeynHiggs [25] and version 3.1.1 of SOFTSUSY [22] leads to a Higgs boson mass of approximately $126 \mathrm{GeV}$ almost independent of $\tilde{m}_{t}[18]$.

In Fig. 2(a) the quantity $\sigma_{t}^{\mathrm{SQCD}}$ is shown as a function of $\tilde{m}_{t}$ at LO, NLO and NNLO (from bottom to top). For each order three curves are shown where the dotted curve corresponds to the SM. The SQCD corrections are included in the dashed and solid line where for the former the soft and hard renormalization scales, $\mu_{s}$ and $\mu_{h}$ have been identified with $M_{h} / 2$ and for the latter $\mu_{s}=M_{h} / 2$ and $\mu_{h}=M_{t}$ has been chosen. 
One observes that the difference between SM and MSSM becomes small for increasing $\tilde{m}_{t}$ which is expected since in this limit the spectrum becomes heavy. However, for smaller values of $\tilde{m}_{t}$ a sizeable effect of the generic SUSY contribution is visible. For example, for $\tilde{m}_{t}=400 \mathrm{GeV}$ a reduction of the SM cross section of about $5 \%$ is observed when including NNLO supersymmetric corrections.

The difference between the dashed and solid line in Fig. 2(a) quantifies the effect of the resummation of $\ln \left(M_{h}^{2} / M_{\text {heavy }}^{2}\right)$ where $M_{\text {heavy }}$ is a heavy mass scale present in the calculation of $C_{1}$. It is negligible for large $\tilde{m}_{t}$, however, for smaller values it can lead to a visible effect.

It is interesting to note that supersymmetric three-loop corrections to $C_{1}$ computed in Ref. $[18,19]$ provide an important contribution to the difference of the solid and dotted curve in Fig. 2(a). In fact, if we choose $\tilde{m}_{t}=400 \mathrm{GeV}$ and identify the three-loop coefficient with the SM one a reduction of only $3 \%$ and not $5 \%$ is observed.

Let us finally present results for $\sigma(p p \rightarrow h+X)$ which include in addition bottom quark contributions up to NLO and furthermore also electroweak corrections. In Fig. 2(b) we show the dependence on $\tilde{m}_{t}$ at LO, NLO and NNLO (from bottom to top). The dotted curves in Fig. 2(b) correspond to the solid ones of Fig. 2(a), i.e. they only include the top-sector contribution. The inclusion of the bottom quark effects at NLO (cf. Eq. (2)) leads to a reduction of about $5 \%$ as shown by the dashed curves. The reduction is basically independent of $\tilde{m}_{t}$ and $\tan \beta .^{3}$ Thus, even for $\tan \beta=20$ the bottom quark effects are small for the considered scenarios and, hence, at NNLO the approximation $m_{b}=0$ is justified. The reduction due to bottom quark effects is to a large extend compensated by the electroweak corrections taken into account multiplicatively as can be seen by the solid line which includes all contributions of Eq. (2).

To conclude this section let us remark that the Higgs boson production cross section within the MSSM is known to the same accuracy as in the SM. The supersymmetric NNLO corrections can effect the production cross section by a few percent in case there is a splitting in the top squark masses by a few hundred $\mathrm{GeV}$ and the overall scale of the spectrum is not too heavy. Such effects are certainly relevant once the experimental precision for the cross section measurement is considerably below $10 \%$, in particular, once there are hints for new particles from direct searches for supersymmetry.

\section{First steps towards $\mathrm{N}^{3}$ LO in the SM}

The cross section for Higgs boson production in gluon fusion has been computed to NLO [26, 27] and NNLO [3, 4, 28-31]. ${ }^{4}$ Nevertheless the contribution from unknown higher orders is estimated to be of the order of $10 \%$ which asks for a $\mathrm{N}^{3} \mathrm{LO}$ calculation. Different groups have started to look into this issue which shall be briefly summarized in the following:

\footnotetext{
${ }^{3}$ The dependence on $\tan \beta$ is studied in Ref. [18].

${ }^{4}$ We refer to the reports of the LHC Higgs cross section working groups for further details and extended lists of references [32, 33].
}

- In Ref. [34] the four-loop corrections to the matching coefficient $C_{1}$ have been constructed from the three-loop decoupling constant for the strong coupling constant with the help of renormalization group methods and a low-energy theorem. In Refs. [35, 36] the result has been confirmed by an explicit calculation of the fourloop decoupling constant.

- The three-loop corrections to the massless Higgs-gluon form factor have been obtained by two independent calculations [37, 38] (see also Ref. [39]).

- The $O(\epsilon)$ contributions to the master integrals needed for the NNLO calculation has been computed in Refs. [6, 40].

- Results for the LO, NLO and NNLO partonic cross sections expanded up to order $\epsilon^{3}, \epsilon^{2}$ and $\epsilon^{1}$, respectively, have been obtained in Ref. [41].

- All contributions from convolutions of partonic cross sections with splitting functions, which are needed for the complete $\mathrm{N}^{3} \mathrm{LO}$ calculation, are provided in Ref. [41]. The results of [41] have been confirmed in Ref. [42].

- Very recently, the full scale dependence of the $\mathrm{N}^{3} \mathrm{LO}$ expression has been constructed in Ref. [42].

- The triple-real contribution to the gluon-induced partonic cross section has been considered in Ref. [43]. In particular, a method has been developed which allows the expansion around the soft limit, i.e. for $y=$ $1-M_{H}^{2} / s \rightarrow 0$. Two expansion terms in $y$ are provided.

- A building block for third-order Higgs boson production is the NNLO correction to the Higgs plus jets production which has been considered in Ref. [44] for the gluongluon channel.

- An approximate $\mathrm{N}^{3} \mathrm{LO}$ expression has been constructed in Ref. [45] from the resummation of soft-gluon and high-energy singularities. (See also Ref. [46] for earlier work along similar lines.)

\section{Acknowledgements}

I would like to thank Maik Höschele, Jens Hoff, Alexey Pak, Takahiro Ueda and Nikolai Zerf for fruitful collaborations on the topics discussed in this contribution. This work was supported by the DFG through the SFB/TR 9 "Computational Particle Physics".

\section{References}

[1] G. Aad et al. [ATLAS Collaboration], Phys. Lett. B 716 (2012) 1 [arXiv:1207.7214 [hep-ex]].

[2] S. Chatrchyan et al. [CMS Collaboration], Phys. Lett. B 716 (2012) 30 [arXiv:1207.7235 [hep-ex]].

[3] R. V. Harlander and K. J. Ozeren, JHEP 0911 (2009) 088 [arXiv:0909.3420 [hep-ph]].

[4] A. Pak, M. Rogal and M. Steinhauser, JHEP 1002 (2010) 025 [arXiv:0911.4662 [hep-ph]]. 
[5] R. V. Harlander, H. Mantler, S. Marzani and K. J. Ozeren, Eur. Phys. J. C 66 (2010) 359 [arXiv:0912.2104 [hep-ph]].

[6] A. Pak, M. Rogal and M. Steinhauser, JHEP 1109 (2011) 088 [arXiv:1107.3391 [hep-ph]].

[7] C. Anastasiou, S. Beerli and A. Daleo, Phys. Rev. Lett. 100 (2008) 241806 [arXiv:0803.3065 [hep-ph]].

[8] R. V. Harlander and M. Steinhauser, Phys. Lett. B 574 (2003) 258 [arXiv:hep-ph/0307346].

[9] R. V. Harlander and M. Steinhauser, JHEP 0409 (2004) 066 [arXiv:hep-ph/0409010].

[10] G. Degrassi and P. Slavich, Nucl. Phys. B 805 (2008) 267 [arXiv:0806.1495 [hep-ph]].

[11] G. Degrassi and P. Slavich, JHEP 1011 (2010) 044 [arXiv:1007.3465 [hep-ph]].

[12] R. V. Harlander, F. Hofmann and H. Mantler, JHEP 1102 (2011) 055 [arXiv:1012.3361 [hep-ph]].

[13] M. Muhlleitner and M. Spira, Nucl. Phys. B 790 (2008) 1 [arXiv:hep-ph/0612254].

[14] R. Bonciani, G. Degrassi and A. Vicini, JHEP 0711 (2007) 095 [arXiv:0709.4227 [hep-ph]].

[15] R. V. Harlander, S. Liebler and H. Mantler, Computer Physics Communications 184 (2013) pp. 1605 [arXiv:1212.3249 [hep-ph]].

[16] R. Harlander and M. Steinhauser, Phys. Rev. D 68 (2003) 111701 [arXiv:hep-ph/0308210].

[17] W. Siegel, Phys. Lett. B 84 (1979) 193.

[18] A. Pak, M. Steinhauser and N. Zerf, JHEP 1209 (2012) 118 [arXiv:1208.1588 [hep-ph]].

[19] A. Kurz, M. Steinhauser and N. Zerf, JHEP 1207 (2012) 138 [arXiv:1206.6675 [hep-ph]].

[20] A. Pak, M. Steinhauser and N. Zerf, Eur. Phys. J. C 71 (2011) 1602 [arXiv:1012.0639 [hep-ph]].

[21] M. S. Carena, S. Heinemeyer, C. E. M. Wagner and G. Weiglein, Eur. Phys. J. C 26 (2003) 601 [hep$\mathrm{ph} / 0202167]$.

[22] B. C. Allanach, Comput. Phys. Commun. 143 (2002) 305 [arXiv:hep-ph/0104145].

[23] R. V. Harlander, P. Kant, L. Mihaila and M. Steinhauser, Phys. Rev. Lett. 100 (2008) 191602 [Phys. Rev. Lett. 101 (2008) 039901] [arXiv:0803.0672 [hep-ph]].

[24] P. Kant, R. V. Harlander, L. Mihaila and M. Steinhauser, JHEP 1008 (2010) 104 [arXiv:1005.5709 [hep$\mathrm{ph}]$.

[25] M. Frank, T. Hahn, S. Heinemeyer, W. Hollik, H. Rzehak and G. Weiglein, JHEP 0702 (2007) 047 [hep-ph/0611326]; http: //www . feynhiggs .de/.
[26] S. Dawson, Nucl. Phys. B 359 (1991) 283.

[27] M. Spira, A. Djouadi, D. Graudenz and P. M. Zerwas, Nucl. Phys. B 453 (1995) 17, arXiv:hep-ph/9504378.

[28] R. V. Harlander and W. B. Kilgore, Phys. Rev. Lett. 88 (2002) 201801, arXiv:hep-ph/0201206.

[29] C. Anastasiou and K. Melnikov, Nucl. Phys. B 646 (2002) 220, arXiv:hep-ph/0207004.

[30] V. Ravindran, J. Smith and W. L. van Neerven, Nucl. Phys. B 665 (2003) 325, arXiv:hep-ph/0302135.

[31] S. Marzani, R. D. Ball, V. Del Duca, S. Forte and A. Vicini, Nucl. Phys. B 800 (2008) 127 [arXiv:0801.2544 [hep-ph]].

[32] S. Dittmaier, C. Mariotti, G. Passarino, R. Tanaka et al., [LHC Higgs Cross Section Working Group Collaboration], arXiv:1101.0593 [hep-ph].

[33] S. Dittmaier, C. Mariotti, G. Passarino, R. Tanaka et al., [LHC Higgs Cross Section Working Group Collaboration], arXiv:1201.3084 [hep-ph].

[34] K. G. Chetyrkin, B. A. Kniehl and M. Steinhauser, Nucl. Phys. B 510 (1998) 61, arXiv:hep-ph/9708255.

[35] Y. Schröder and M. Steinhauser, JHEP 0601 (2006) 051, arXiv:hep-ph/0512058.

[36] K. G. Chetyrkin, J. H. Kühn and C. Sturm, Nucl. Phys. B 744 (2006) 121, arXiv:hep-ph/0512060.

[37] P. A. Baikov, K. G. Chetyrkin, A. V. Smirnov, V. A. Smirnov and M. Steinhauser, Phys. Rev. Lett. 102 (2009) 212002 [arXiv:0902.3519 [hep-ph]].

[38] T. Gehrmann, E. W. N. Glover, T. Huber, N. Ikizlerli and C. Studerus, JHEP 1006 (2010) 094 [arXiv:1004.3653 [hep-ph]].

[39] R. N. Lee, A. V. Smirnov and V. A. Smirnov, JHEP 1004 (2010) 020 [arXiv:1001.2887 [hep-ph]].

[40] C. Anastasiou, S. Buehler, C. Duhr and F. Herzog, JHEP 1211 (2012) 062 [arXiv:1208.3130 [hep-ph]].

[41] M. Höschele, J. Hoff, A. Pak, M. Steinhauser and T. Ueda, Phys. Lett. B 721 (2013) 244 [arXiv:1211.6559 [hep-ph]].

[42] S. Buehler and A. Lazopoulos, arXiv:1306.2223 [hep-ph].

[43] C. Anastasiou, C. Duhr, F. Dulat and B. Mistlberger, arXiv:1302.4379 [hep-ph].

[44] R. Boughezal, F. Caola, K. Melnikov, F. Petriello and M. Schulze, arXiv:1302.6216 [hep-ph].

[45] R. D. Ball, M. Bonvini, S. Forte, S. Marzani and G. Ridolfi, arXiv:1303.3590 [hep-ph].

[46] S. Moch and A. Vogt, Phys. Lett. B 631 (2005) 48 [hep-ph/0508265]. 